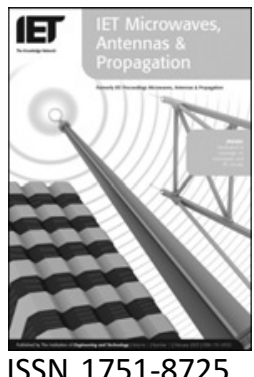

\title{
Analysis of the left-handed corrugated circular waveguide
}

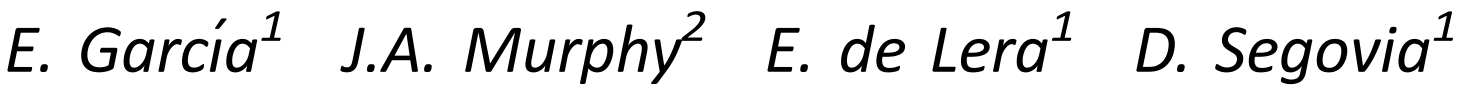

\author{
${ }^{1}$ Departmento of Teoría de la Señal y Comunicaciones, Universidad Carlos III de Madrid, Spain \\ ${ }^{2}$ Department of Experimental Physics, National University of Ireland, Maynooth, Co., Kildare, Ireland \\ E-mail: quique@gr.ssr.upm.es
}

\begin{abstract}
Infinite circular corrugated waveguide is analysed to investigate its ability to support modes with backward wave behaviour. Such waveguides provide an alternative structure, easier to manufacture than those already reported based on rectangular symmetry with corrugated walls or filled with frequency selective surfaces. The corrugations if sufficiently deep provide a guiding structure with the required series capacitance and shunt inductance to allow left-handed propagation within some frequency bands. These backward waves are analysed using the surface impedance model of propagation in corrugated waveguides to predict their properties. Interpreting the physical meaning of the analysis, the authors discuss how backward waves are related to resonances in corrugated structures. The relationship between power flows in the guide and the behaviour of the group velocity for such guides is shown. A full wave simulator is also applied to validate these results and the case of a dielectric filled waveguide is considered showing the improved ability to support left-handed modes. The authors present the results of a parametric study of how left-handed propagation depends on the corrugation depth. Potential applications of backward waves in corrugated circular waveguides are proposed.
\end{abstract}

\section{Introduction}

Metmaterial structures for microwave and antenna devices have been amply introduced and studied in recent years $[1,2]$. These are structures that support left-handed propagation in addition to conventional right-handed propagation. Several studies have been made of transmission lines with left-handed behaviour [3, 4]. Principal advances and applications using metamaterials in transmission lines include improved response and more compact design. Among guided-wave structures, waveguides are characterised by their low loss, high-power handling capability and the absence of leakage and other extraneous phenomena due to their closed geometries. The first papers related with such structures examined the lefthanded propagation of backward waves $[5,6]$. The propagation of backward waves in rectangular waveguides with printed split-ring resonators was investigated. In addition, corrugated waveguides with dielectric-filled corrugations were also analysed using an equivalent transmission line model $[7,8]$. Furthermore, modal and dispersion characteristics of rectangular waveguides with dielectric-filled corrugations were studied in recent papers. Another study of backward wave propagation in a waveguide based on periodic structures loaded with various frequency selective surface (FSS) strip layers is described in detail in [3-9]. Therefore as reported thus far in the literature, backward wave propagation behaviour of a waveguide is achieved both by means of periodic strip FSS layers and also by making use of corrugations filled with dielectric. However, all of these studies have been always based on rectangular waveguides.

In this paper, we report on our study of the modal and dispersion characteristics of circular corrugated waveguide to probe its ability to support backward waves, that is, waves that exhibit phase advance in the direction of propagation. Propagation in circular corrugated waveguide has been extensively discussed by Clarricoats and Olver $[10,11]$. Corrugated waveguides have been used in horn antenna applications, where the corrugations serve as a high-impedance surface required to support hybrid modes 
that improve the radiation characteristics. A new function for the corrugated surface may, however, be sought to support backward waves in the waveguide. For left-handed waves to propagate, the guiding structure should provide series capacitance and shunt inductance within some frequency ranges. Corrugations introduce such an effect provided they are sufficiently deep.

We aim to demonstrate the ability of circular corrugated waveguide to support backward waves of a simple form. The principal advantages compared with a rectangular waveguide are the easier manufacture of the circular waveguide, the availability of two polarisations for multiple applications and miniaturisation of systems and devices. In addition, we also suggest other significant and powerful applications such as bandpass filtering in waveguide-fed quasi-optical systems and negative refractive index-based microwave plate lenses.

The main theoretical approach we have taken for the analysis of backward waves in corrugated circular waveguide is the so-called surface impedance method, as described by Clarricoats. In this approach, we consider the waveguide as having its corrugated surface replaced by a wall of uniform non-isotropic impedance (with different impedances in the transverse and axial directions) [12]. The analysis is relatively straightforward to implement and complemented by the qualitative insight given by mode-matching techniques $[10,11]$. It allows us to predict the approximate frequency bands for backward wave behaviour for waveguides with different relative slot depths. In the modematching technique, the corrugated waveguide structure is regarded as a sequence of cylindrical waveguide segments with the radius stepping between the top and bottom of the corrugation slots $[11-15]$. We then go on present the results of a full-wave simulation based on CST software [16] (based on the FDTD method). This allows us to analyse more complex structures such as waveguides with dielectrically filled grooves.

The theoretical analysis of the corrugated circular waveguide based on the surface impedance model is presented in Section 2. Dispersion diagram characteristics and power-flow distributions are discussed. In Section 3, we present the results of computational modelling, including the example of waveguide with the corrugations filled with high dielectric permittivity. We present a parametric study of this case. In Section 4 we discuss potential applications of corrugated circular waveguide as a backward wave-supporting structure, and conclusions and future work are discussed in Section 5.

\section{Analytical approach}

From an analytical viewpoint, we can most easily study backward wave propagation in corrugated waveguides by considering the surface impedance technique as described by Clarricoats and Olver [10, 11], in which the corrugations are represented by a continuous non-isotropic impedance surface bounding an inner region of radius $r_{1}$. (Fig. 1) This approach assumes many corrugations per wavelength $(>4)$ and narrow slots, so that only a single non-propagating TM mode is capable of existing in the slot (of depth $r_{0}-r_{1}$ ). The propagating hybrid waveguide modes in the inner guide (of depth $r<r_{1}$ ) can be regarded as a mixture of TE and TM fields, with the $z$ components of the electric and magnetic fields

$$
E_{z}(r, \phi)=a_{m} J_{m}(K r) \cos m \phi \exp \mathrm{j}(\omega t-\beta z)
$$

and

$$
H_{z}(r, \phi)=a_{m} y_{0} \bar{\Lambda} J_{m}(K r) \sin m \phi \exp (\omega t-\mathrm{j} \beta z)
$$

where $y_{0}$ is the admittance of free space, $\beta$ the propagation coefficient with $K^{2}=k^{2}-\beta^{2}$ (the usual waveguide equation), $k=\omega / c$ and $\bar{\Lambda}$ the so-called normalised hybrid factor. The transverse fields can be derived from the $z$-component of the fields in the usual way [17]

$$
\begin{aligned}
& E_{r}(r, \phi)=-\frac{\mathrm{j}}{K^{2}}\left(\beta \frac{\partial E_{z}}{\partial r}+\frac{\omega \mu_{0}}{r} \frac{\partial H_{z}}{\partial \phi}\right) \\
& E_{\phi}(r, \phi)=-\frac{\mathrm{j}}{K^{2}}\left(\frac{\beta}{r} \frac{\partial E_{z}}{\partial \phi}-\omega \mu_{0} \frac{\partial H_{z}}{\partial r}\right) \\
& H_{r}(r, \phi)=+\frac{\mathrm{j}}{K^{2}}\left(\frac{\omega \varepsilon_{0}}{r} \frac{\partial E_{z}}{\partial \phi}-\beta \frac{\partial H_{z}}{\partial r}\right) \\
& H_{\phi}(r, \phi)=-\frac{\mathrm{j}}{K^{2}}\left(\omega \varepsilon_{0} \frac{\partial E_{z}}{\partial r}-\frac{\beta}{r} \frac{\partial H_{z}}{\partial \phi}\right)
\end{aligned}
$$

so that, for example

$$
\begin{aligned}
E_{\phi}(r, \phi)= & \frac{\mathrm{j} a_{m}}{K^{2}}\left(\frac{m \beta}{r} J_{m}(K r)+k \bar{\Lambda} K J_{m}^{\prime}(K r)\right) \\
& \times \sin m \phi \operatorname{expj}(\omega t-\beta z)
\end{aligned}
$$

The necessary requirement that the $\phi$ component of the electric field be zero $\left(E_{\varphi}=0\right)$ at the corrugations yields the following relationship between $\bar{\Lambda}$ and $\beta$

$$
\bar{\Lambda}=-\frac{m \beta J_{m}\left(K r_{1}\right)}{k K r_{1} J_{m}^{\prime}\left(K r_{1}\right)}=-\frac{m \bar{\beta}}{F_{m}\left(K r_{1}\right)}
$$

where $F_{m}(x)=x J_{m}^{\prime}(x) / J_{m}(x)$ and where $\bar{\beta}=\beta / k$.

As discussed in Clarricoats [10] in order to derive the dispersion relationships for the propagating modes in the waveguide relating $k$ and $\beta$, we match at $r=r_{1}$ (the radius

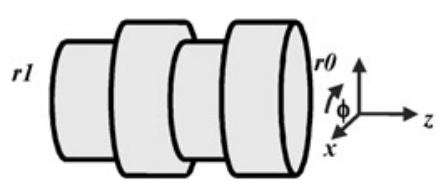

Figure 1 Definition of the waveguide structure 
of the inner guide) the admittance of the hybrid mode in the inner region of the guide given by $Y=\left(H_{\varphi}\right)$ $\left.E_{z}\right)=-j y_{0}(k / K)^{2}\left[F m\left(K r_{1}\right)-m \beta^{2} / F m\left(K r_{1}\right)\right]$ to the surface admittance of the lowest order $\mathrm{TM}_{m}$ in the slot, $Y=-j y_{o} S\left(k r_{1}, k r_{0}\right)$, where $S_{m}(x, y)$ is given by

$$
S_{m}(x, y)=x \frac{J_{m}^{\prime}(x) Y_{m}(y)-J_{m}(y) Y_{m}^{\prime}(x)}{J_{m}(x) Y_{m}(y)-J_{m}(y) Y_{m}(x)}
$$

This yields the characteristic equation for $\beta$

$$
F_{m}\left(K r_{1}\right)-\frac{(m \bar{\beta})^{2}}{F_{m}\left(K r_{1}\right)}=\left(\frac{K r_{1}}{k r_{1}}\right)^{2} S_{m}\left(k r_{1}, k r_{0}\right)
$$

It is clear from the work of Clarricoats that for sufficiently deep corrugations, dispersion curves with negative slopes $(\mathrm{d} \beta / \mathrm{d} k)$ can exist, these in general corresponding to the lowest order mode of the different families of modes defined by their azimuthal symmetries. For example, the dispersion curves for the two lowest order modes with azimuthally symmetry defined by $m=1$ and for the case of $r_{1} / r_{0}=0.48$ are shown in Fig. 2.

Clearly, the $\mathrm{EH}_{11}$ is a backward wave in the sense that the group velocity $(\mathrm{d} k / \mathrm{d} \beta) \cdot c$ is negative, whereas the phase velocity $(k / \beta) \cdot c$ is positive (or vice versa). In fact, for values of $r_{1} / r_{0}$ less than about 0.6 , the lowest order mode $\mathrm{EH}_{11}$ mode has a significant section of the dispersion curve $(k, \beta)$ with a negative slope $\mathrm{d} \beta / \mathrm{d} k$ implying backward wave behaviour over useful finite bandwidths (Fig. 3). Results are coincident with those presented in $[12,13]$.

The strongest backward wave effects occur for $r_{1} / r_{0} \leq 0.48$ approximately. It is noteworthy that the nature of the $\mathrm{EH}_{11}$ mode changes at cut-on $(\beta=0)$ around this value, from pure TM for $r_{1} / r_{0}<0.480$ with $k r_{1}=1.8412$, to pure hybrid with $\bar{\Lambda}=-1$ for $r_{1} / r_{0}=0.480$ and pure TE for $r_{1} / r_{0}>$ 0.480 with $k r_{1}=3.8317$. This is because as noted by Clarricoats [10] there are two cases for which the

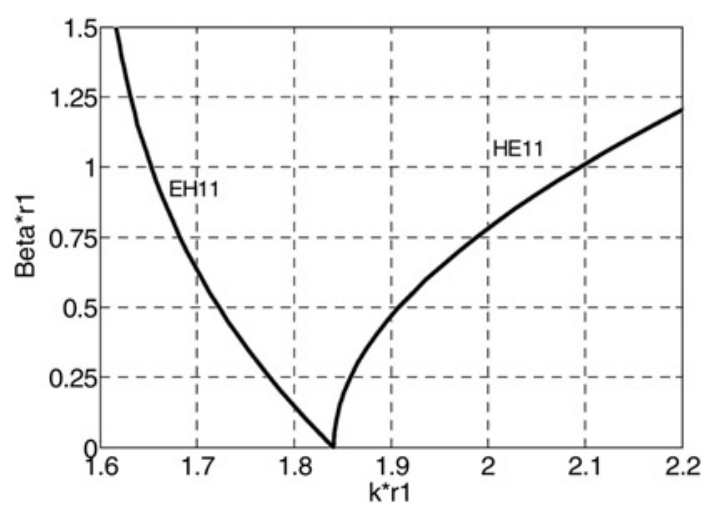

Figure 2 Dispersion diagrams for $H E_{11}$ and $E H_{11}$ modes
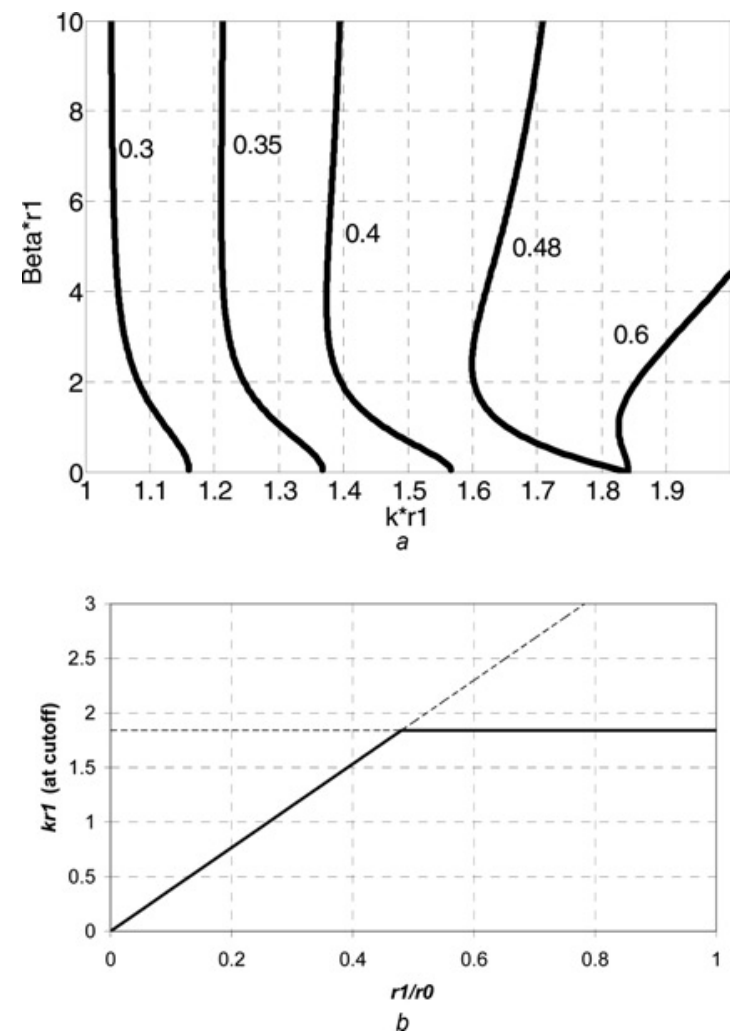

Figure 3 Dispersion curves for $E_{11}$ mode and cut-off/on $k r_{1}$ for $\mathrm{EH}_{11}$ mode

a Dispersion curves for $\mathrm{EH}_{11}$ mode for several values of $r_{1} / r_{0}=0.30,0.35,0.40,0.48 .0 .60$

$b$ Cut-off/on $k r_{1}$ for $\mathrm{EH}_{11}$ mode (bold curve) as a function of $r_{1} / r_{0}$ Dashed line represents the cut-off for the $\mathrm{HE}_{11}$ mode

characteristic equation is satisfied with $\beta=0$, either $J_{1}^{\prime}\left(k r_{1}\right)=$ 0 (which corresponds to pure TE mode in the inner guide at cut-off/on with $k r_{1}=1.8411$ ) or $J_{1}\left(k r_{0}\right)=0$ (which corresponds to pure TM in the inner guide). These two cases represent the cut-off/on $k r_{1}$ of either the $\mathrm{EH}_{11}$ or the $H E_{11}$ mode, with the $E H_{11}$ cut-off/on occuring at the lower value of the two possible cases of $k r_{1}$ for which $\beta=0$. As illustrated in Fig. 4, for $r_{1} / r_{0}<0.48$, the $\mathrm{EH}_{11}$ cut-on varies linearly with $k r_{1}$ (TM in the inner guide), whereas for $r_{1} / r_{0}>$ 0.48 , it is a constant at $k r_{1}=1.8411$ ( $\mathrm{TE}$ in the inner guide). When $r_{1} / r_{0}=0.48$, both conditions are satisfied and cut-off of both modes occurs at the same value of $k r_{1}$.

In fact, to understand physically what is happening in the guide $\left(r<r_{1}\right)$, it is illuminating to consider the power flows and the behaviour of the Poynting vector with $r$. Clearly, for a backward wave, we expect that the power flow will be opposite in direction to the phase velocity of the hybrid mode. In other words, the flux of the pointing vector $\boldsymbol{S}$ over the guide cross-section will be opposite in direction to that of $\boldsymbol{\beta}$ (the propagation wavevector for the mode along the guide at least for some values of $r$ ). For the=case where $m=1$, the Poynting vector, $\boldsymbol{S}_{z}=$ $\left(\boldsymbol{E} \times \boldsymbol{H}^{*}\right)_{z}=E_{x} H_{y}^{*}-E_{y} H_{x}^{*}$ can be written (after some 


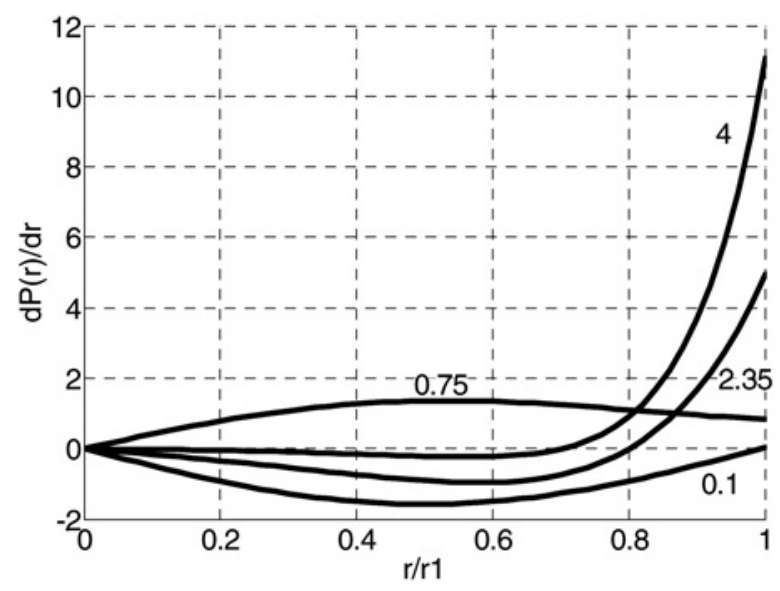

Figure 4 Plot showing the power flow per annulus $d P(r) / d r$ for various values of $\beta r_{1}$, showing backward flow for low values and forward flow along corrugated walls for high values $\left(r_{1} / r_{0}=0.48\right)$

algebraic manipulations) as

$$
\begin{aligned}
\boldsymbol{S}_{z}= & y_{0}\left(a_{1} k / 2|K|\right)^{2}\left((\bar{\beta}+\bar{\Lambda})(1+\bar{\beta} \bar{\Lambda}) J_{0}^{2}(K r)\right. \\
& -(\bar{\Lambda}-\bar{\beta})(1-\bar{\beta} \bar{\Lambda}) J_{2}^{2}(K r)-[(\bar{\Lambda}+\bar{\beta})(1-\bar{\beta} \bar{\Lambda}) \\
& \left.+(\bar{\Lambda}-\bar{\beta})(1+\bar{\beta} \bar{\Lambda})]\left[J_{0}(K r) J_{2}(K r) \cos 2 \phi\right]\right)
\end{aligned}
$$

We divide the waveguide cross-section into infinitesimal concentric annuli of radii $r$ and width $\mathrm{d} r$ and integrate the Poynting vector over the area of the annulus to compare how power flow $\mathrm{d} P$ in an annulus of width $\mathrm{d} r$ varies as a function of $r$. Clearly, $\mathrm{d} P(r)$ is given by

$$
\begin{aligned}
\mathrm{d} P(r)= & {\left[\int_{0}^{2 \pi} \boldsymbol{S}_{z}(r, \phi) \mathrm{d} \phi\right] r \mathrm{~d} r } \\
= & y_{0}\left(\frac{a_{1}}{2} \frac{k}{|K|}\right)^{2}\left(\bar{\beta}\left(1+\bar{\Lambda}^{2}\right)\left[J_{0}^{2}(K r)+J_{2}^{2}(K r)\right]\right. \\
& \left.+\bar{\Lambda}\left(1+\bar{\beta}^{2}\right)\left[J_{0}^{2}(K r)-J_{2}^{2}(K r)\right]\right) 2 \pi r \mathrm{~d} r
\end{aligned}
$$

If for different cases of $r_{1} / r_{0}<0.6$, we now consider the sign of $\mathrm{d} P(r)$ as $\beta$ is varied along the dispersion curve $\left(k r_{1}, \beta r_{1}\right)$, we find that the power flow is along the $-z$ direction for at least some parts of the waveguide crosssection $\left(r<r_{1}\right)$.

We will first consider power flows for the case $r_{1} / r_{0}=0.48$ in this regard. At $\beta=0$ (cut-on/off), the $\mathrm{EH}_{11}$ mode is pure hybrid with $\bar{\Lambda}=-1$. This also coincides with the admittance of the corrugation slot being zero since $S_{m}\left(k r r_{1}\right.$, $\left.k r_{0}\right)=0$, implying the slot is 'resonant' with a standing wave in the radial direction since $H_{\varphi}=0$ at $r=r_{1}$ and $r_{0}$. We see that for small values of $\beta r_{1} \ll 1$, since $\bar{\Lambda}$ must still be given by $\bar{\Lambda} \simeq-1$, then $\mathrm{d} P(r)$, the power flow in an annulus of width $\mathrm{d} r$, must be negative since for all $r<r_{1}$ (implying a backward flowing wave)

$$
\begin{aligned}
\mathrm{d} P(r) & =\left[\int_{0}^{2 \pi} S_{z}(r, \phi) \mathrm{d} \phi\right] r \mathrm{~d} r \\
& \simeq(-1) y_{0}\left(\frac{a_{1}}{2} \frac{k}{|K|}\right)^{2}\left(J_{0}^{2}(k r)-J_{2}^{2}(k r)\right) 2 \pi r \mathrm{~d} r<0
\end{aligned}
$$

This is because $J_{0}^{2}(k r)-J_{2}^{2}(k r)=J_{1}(k r) J_{1}^{\prime}(k r) / 4 \geq 0$, as long as $k r_{1}<1.8414$. Thus, the wave has to be left handed for low values of $\beta$. Since $\mathrm{d} P(\mathrm{r})$ represents the power flow through an annulus of width $\mathrm{d} r$ and is clearly proportional to $\mathrm{d} r$, we therefore plot $\mathrm{d} P(r) / \mathrm{d} r$ (the power flow through an annular ring of width $\mathrm{d} r$ divided by $\mathrm{d} r$ ) as a function of $r / r_{1}$ in Fig. 4. Thus, for example at point $1,\left(k r_{i}, \beta r_{i}\right)=$ $(1.812,0.100)$, we see that $\mathrm{d} P(r) / \mathrm{d} r$ is essentially negative for all values of $r$ (Fig. 4).

As $\beta r_{i}$ increases, we find that the pattern of $\mathrm{d} P(r) / \mathrm{d} r$ changes. Thus, there is some power flow in the positive $z$ direction (with $\mathrm{d} P(r) / \mathrm{d} r>0$ ) close to walls. Note that for the forward $\mathrm{HE}$ wave we obtain the more 'normal' behaviour expected (with $\mathrm{d} P(r) / \mathrm{d} r>0$ ) for all values of $r$. The case of $\left(k r_{i}, \beta r_{i}\right)=(1.9881,0.75)$ is also shown in Fig. 4. As $\beta$ increases further and we approach the inflection point, the integrated Poynting vector crosssection (i.e. total power flow in the guide) tends to zero

$$
\boldsymbol{P}=\int_{0}^{r_{1}} \int_{0}^{2 \pi} S_{z}(r, \phi) r \mathrm{~d} r \mathrm{~d} \phi=\int_{0}^{r_{1}} \mathrm{~d} P(r) \rightarrow 0
$$

and there is a balance between power flowing 'backward' along the central region of the guide and 'forward' close to the corrugated walls, again as illustrated in Fig. 4. For higher values of $\beta r_{i}>2.3$, more power flows in the forward direction (close to the corrugated walls) and the mode becomes a forward mode (Fig. 4). Thus, for $\left(k r_{i}, \beta r_{i}\right)=$ $(1.6205,4.00)$, power flow is predominantly close to the corrugated walls and in the forward direction only and the mode becomes a conventional forward one.

For $r_{1} / r_{0}>0.48$, the mode is pure TE at cut-on and the backward flow of power becomes less pronounced (e.g. $r_{1} / r_{0}=0.60$, as shown in Fig. 5). There is already a forward component of the total power in the guide close to the corrugated walls even at cut-off/on for $\beta r_{1}=0$, and as $\beta r_{1}$ increases, balance is quickly reached between the total power flowing in the reverse and forward directions. Again this balance occurs at the value of $\left(k r_{i}, \beta r_{i}\right)$, for which the group velocity $\mathrm{d} \beta / \mathrm{d} k$ is zero (point of inflection). The mode is only backward over a rather small bandwidth.

The situation is more interesting for cases where $r_{1} / r_{0}<$ 0.48 as the backward wave regime in the guide has a bigger bandwidth. The backward mode is then pure TM $(\bar{\Lambda}=\infty)$ at cut-on $(\beta=0)$ and the region of the guide close to the corrugations now has a negative non-zero power flow $\mathrm{d} P(r) / \mathrm{d} r$. As we move upwards along the 


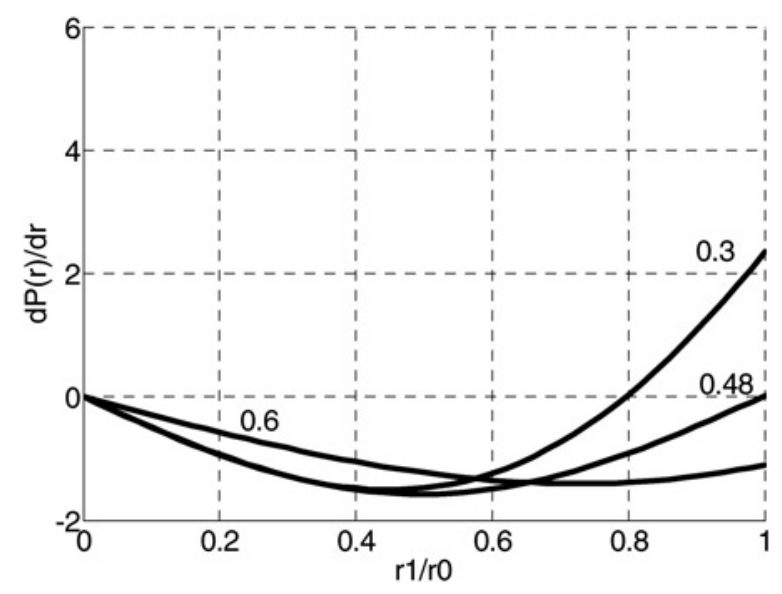

Figure 5 Plot showing the power flow per annulus $d P(r) / d r$ close to cut-on/off $\beta r_{1} \geq 0$ for various values of $r_{1} /$ $r_{0}=0.4,0.48,0.6$

dispersion curve (as $\beta r_{1}$ increases), and provided the corrugations are not too deep $\left(0.48<r_{1} / r_{0}<0.35\right.$, approximately), we reach a point where the admittance of the walls shrinks to zero, $S_{m}\left(k r r_{1}, k r_{0}\right)=0$, again implying the slot is 'resonant' with a standing wave in the radial direction with $H_{\varphi}=0$ at $r=r_{1}$ and $r_{0}$. At this point, $\mathrm{d} P(r) / \mathrm{d} r$ is now zero at $r=r_{1}$. Then as $\beta r_{1}$ grows further along the dispersion curve power flow $\mathrm{d} P(r) / \mathrm{d} r$ in the positive direction for the region close to the corrugations sets in, and eventually balance is reached between the total power flowing in the forward and reverse directions, so that total power flow is zero. This point again coincides with the group velocity also being zero. In general, we see that the group velocity reaching zero (inflection point on the dispersion curve) coincides with the total power in the guide (integrated Poynting vector). See Fig. 6 for example cases of $r_{1} / r_{0}=0.48,0.60$.

For very deep corrugations $\left(r_{1} / r_{0}=0.30\right.$ or less $)$, however, the power flow remains negative and the point on the dispersion

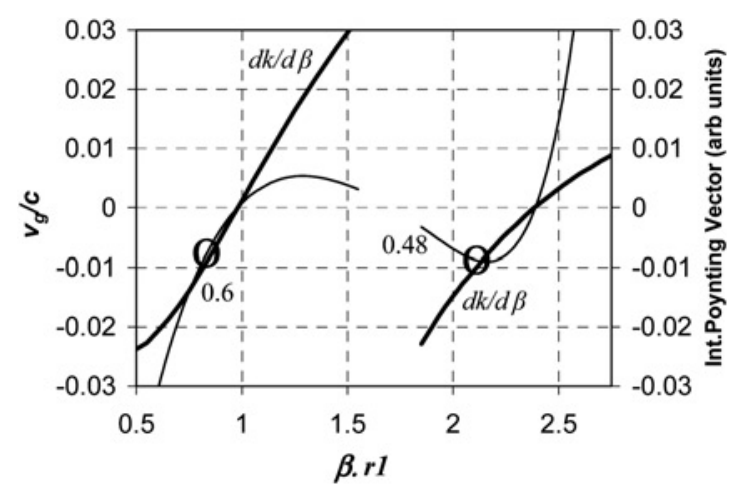

Figure 6 Plot of $d k / d \beta$ (bold curves) and total integrated Poynting vector (light lines) in relative units, as a function of $\beta r_{1}$ for the two cases $r_{1} / r_{0}=0.48$ (right-hand curves) and 0.60 (left-hand curves)

Both curves change sign at the same value of $\left(k r_{1}, \beta r_{1}\right)$ curve where the admittance is zero, $S_{m}\left(k r_{1}, k r_{0}\right)=0$ is only reached as $\beta r_{1} \rightarrow \infty$. In fact, the power flow also becomes concentrated close to the corrugated walls as $\beta r_{1} \rightarrow \infty$, although now in the backward direction, the field flux drops sharply to zero right at the walls. In this section, we have only considered the case of $m=1$. Similar behaviour exists for higher values of $m$, but with the backward wave behaviour at higher values of $k r_{1}$. The symmetry of these modes is, of course, more complex. From a scattering matrix viewpoint, we regard the waveguide as made up of a sequence of wide and narrow circular waveguide segments.

Conventional circular waveguide modes propagate in each segment in both the forward and backward directions. Modes may also be evanescent. At the junction between two segments, the modes are scattered by the discontinuity. The mode-matching technique allows this scattering to be computed through the conservation of complex power. This is desribed in detail by Olver [11].

What is unusual for the case of the backward wave mode for $m=1$ and $r_{1} / r_{0}=0.48$, for example, is that for $k r_{1}<$ 1.8414 no propagating waveguide mode can exist in the narrow waveguide segments. Yet for the band range of the $\mathrm{EH}_{11}$ mode in fact we have $k r_{1}<1.8414$. On the other hand, of course, the wider waveguide segments (coinciding with the slots) can support a propagating $\mathrm{TE}_{11}$ mode, otherwise there would be no propagation at all. Furthermore, in order to satisfy the boundary conditions, a significant number of higher-order evanescent modes are also required in both the narrow and wide guide sections. In fact, the wide segments (with the deep slots) act like cavities. The system therefore behaves like a guide consisting of coupled waveguide cavities (in which both propagating and evanescent modes exist) connected by short sections in which all modes are evanescent. In this case, clearly a complex multi-moded standing wave structure will build up in the cavities and we see qualitatively that the classic set up for backward wave behaviour exists, in that the system consists of a series of coupled oscillators. The backward wave behaviour only sets in therefore when there is a necessary strong scattering between the modes.

\section{Computational results}

We present the results of a corrugated circular waveguide obtained with a full-wave simulation from CST software [18]. In Fig. 7, we show the universal dispersion diagram for the first seven modes (some of which are degenerated) for $r_{1} / r_{0}=0.5$ close to the value of 0.48 considered in Section 2 .

As we can see from the slopes of the dispersion curves, it is possible to obtain modes that can support both forward and backward waves. The passbands for these modes as well as the stopbands of the waveguide are obvious. Also, in Fig. 8, we show the electric and magnetic energy densities for the first 


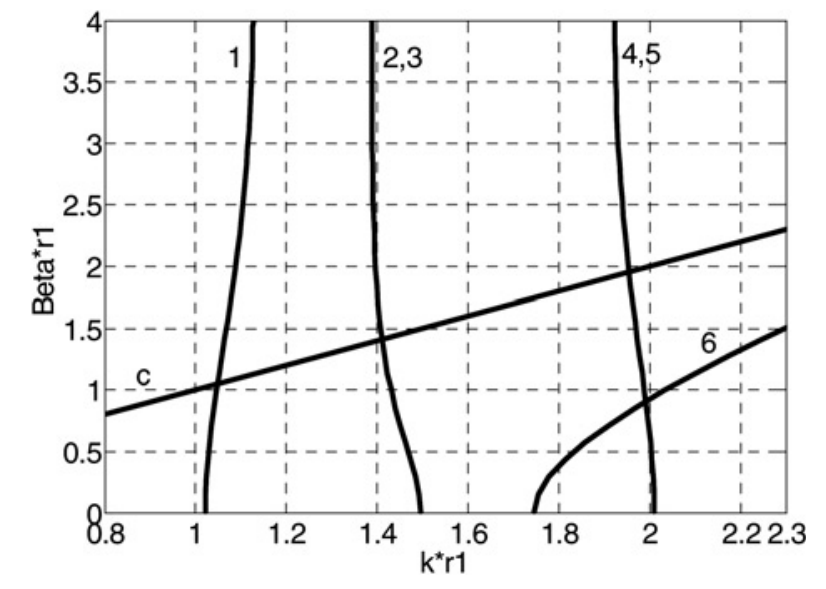

Figure 7 Dispersion diagram of an unfilled circular corrugated waveguide with $r_{1} / r_{0}=0.5$

Degenerate modes correspond to: mode $1 \rightarrow E 01$, modes 2,3 $\rightarrow \mathrm{EH}_{11}$, modes $4,5 \rightarrow \mathrm{EH}_{21}$ and modes $6,7 \rightarrow \mathrm{HE}_{11}$

left-handed mode $\mathrm{EH}_{11}$, which are the degenerate modes 2 and 3 of Fig. 7 (i.e. the two polarisations of $\mathrm{EH}_{11}$ ). As developed analytically in the previous section, for the case $m=1 \quad\left(\mathrm{EH}_{11}\right)$, there exists for a range of ratios, approximately defined by $0.35<r_{1} / r_{0}<0.5$, a resonance in the structure (at some value of $k r_{1}$ ) corresponding to a radial standing wave in the waveguide slot.

In Fig. 9, we present a parametric study of the dispersion diagram for the second mode $\left(\mathrm{EH}_{11}\right)$ of the corrugated circular waveguide. This mode is left handed but when the

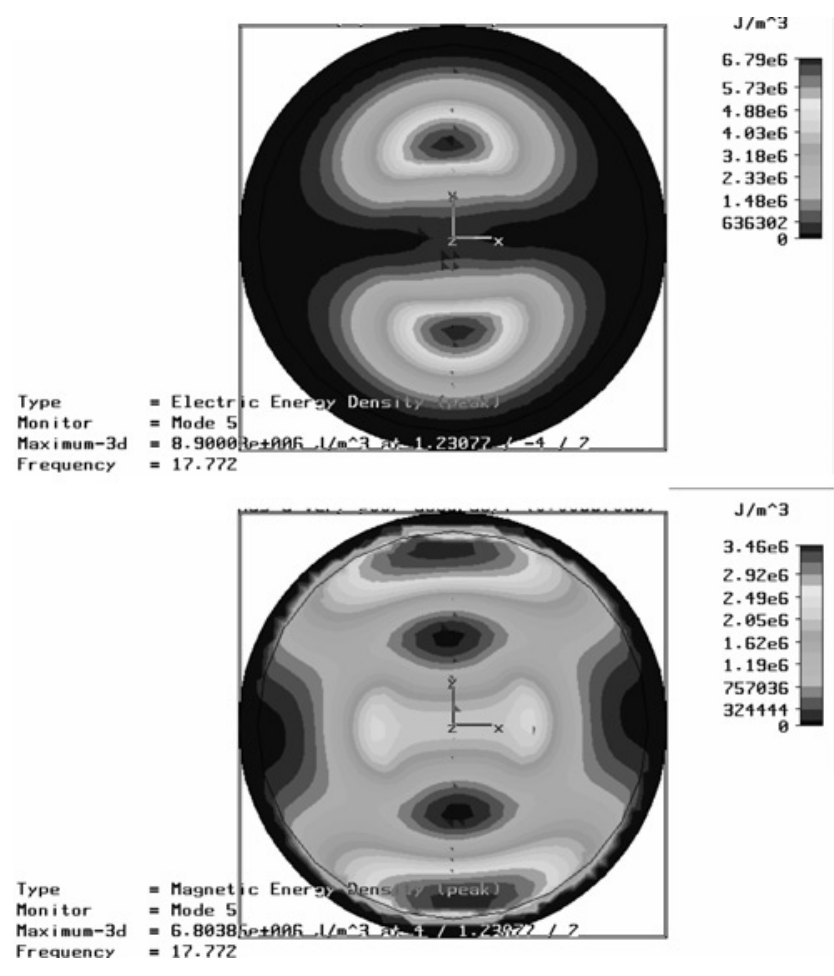

Figure 8 Electric and magnetic energy densities for $\mathrm{EH}_{11}$

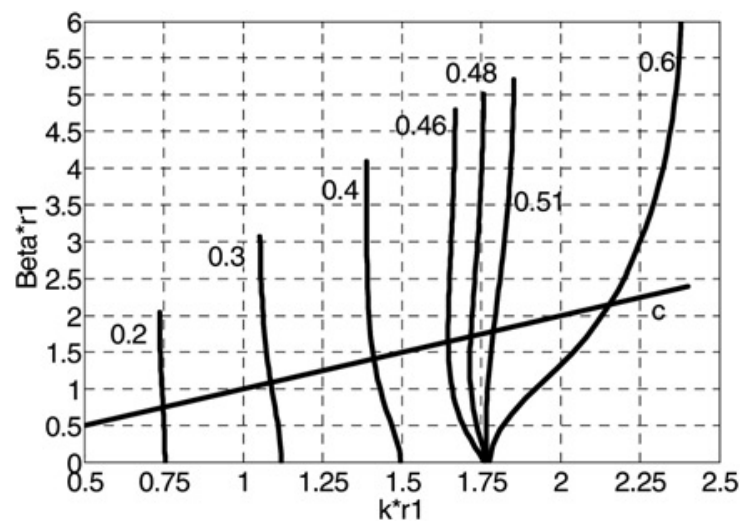

Figure 9 Parametric study of the mode $\mathrm{EH}_{11}$ for different values of the ratio $r_{1} / r_{0}$

ratio $r_{1} / r_{0}$ has a value greater than 0.5 , the propagation of the mode changes to being predominantly forward. Clearly, for a range of ratios $r_{1} / r_{0}$ between 0.35 and 0.5 , we obtain potential useful bandwidths of the order of $10 \%$.

We study also the behaviour of a corrugated circular waveguide with the corrugations filled with dielectric as described in Fig. 10. Fig. 11 shows the dispersion diagram for the first 10 modes of a corrugated circular waveguide (including degenerate modes) with $r_{0}$ equal to $10 \mathrm{~mm}$ and with the depth of the corrugation $r_{0}-r_{1}$ of $4 \mathrm{~mm}$ (i.e. inner radius $r_{1}=6 \mathrm{~mm}$ and $r_{0} / r_{1}=0.6$ ). The width of the corrugation is $1 \mathrm{~mm}$. The dielectric permittivity of the filled corrugation has a value of $\varepsilon_{\mathrm{r} 2}=10$ with the central guide empty $\varepsilon_{\mathrm{r} 1}=1$. As we can see, there exists a backward wave in all modes except the first $\left(\mathrm{E}_{01}\right)$ [10]. Thus, the application of a corrugated waveguide as a composite metamaterial guided-wave structure is achieved. The dispersion diagram obtained through the analysis of the periodic structure exhibits forward and backward wave passbands. The bandwidth of the latter can be controlled by tuning the corrugations parameters, such as corrugation period, depth and width as well as the waveguide radius.

As we can see from the dispersion diagram, there exist passbands and stopbands in the waveguide. Therefore in fact, it is possible to use left-handed mode propagation in a bandpass with no propagation of other modes. For example, for the first two left-handed modes from Fig. 12, we can see that modes 2 and 3 are degenerated. In Figs. 12 and 13 , we present the electric energy densities in the

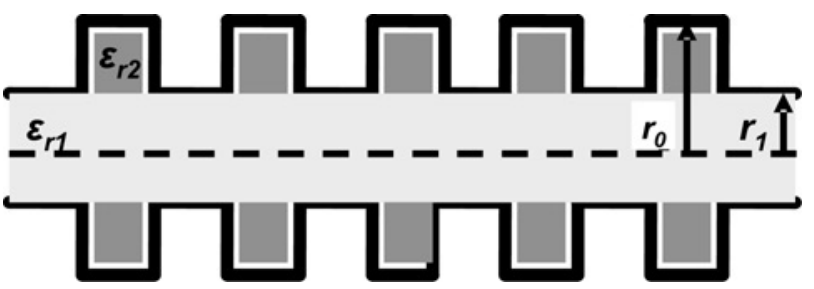

Figure 10 Definition of the waveguide structure 


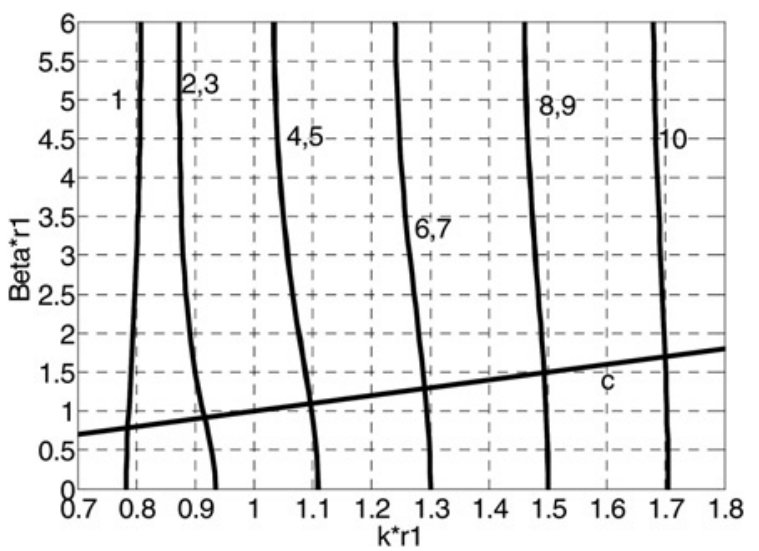

Figure 11 Dispersion diagram of a filled circular corrugated waveguide with $r_{1} / r_{0}=0.6$

Degenerate modes correspond to: mode $1 \rightarrow E_{01}$, modes $2,3 \rightarrow \mathrm{EH}_{11}$, modes $4,5 \rightarrow \mathrm{EH}_{21}$, modes $6,7 \rightarrow \mathrm{EH}_{31}$, modes $8,9 \rightarrow \mathrm{EH}_{4,1}$ and modes $10,11 \rightarrow \mathrm{EH}_{5,1}$

transverse cross-section of the waveguide for the two polarisations of the two modes $\mathrm{EH}_{11}$ and $\mathrm{EH}_{21}$.

If we increase the ratio $r_{1} / r_{0}$ (by increasing $r_{1}$ ), a similar phenomenon occurs to that of the circular waveguide with no dielectric in the grooves. In Fig. 14, we present the results of the first two degenerate left-handed modes $\left(\mathrm{EH}_{11}\right)$ for a value of $r_{0}$ equal to $10 \mathrm{~mm}$ and $r_{1}$ varying from 5 to $8 \mathrm{~mm}$ (so that the depth of the corrugations
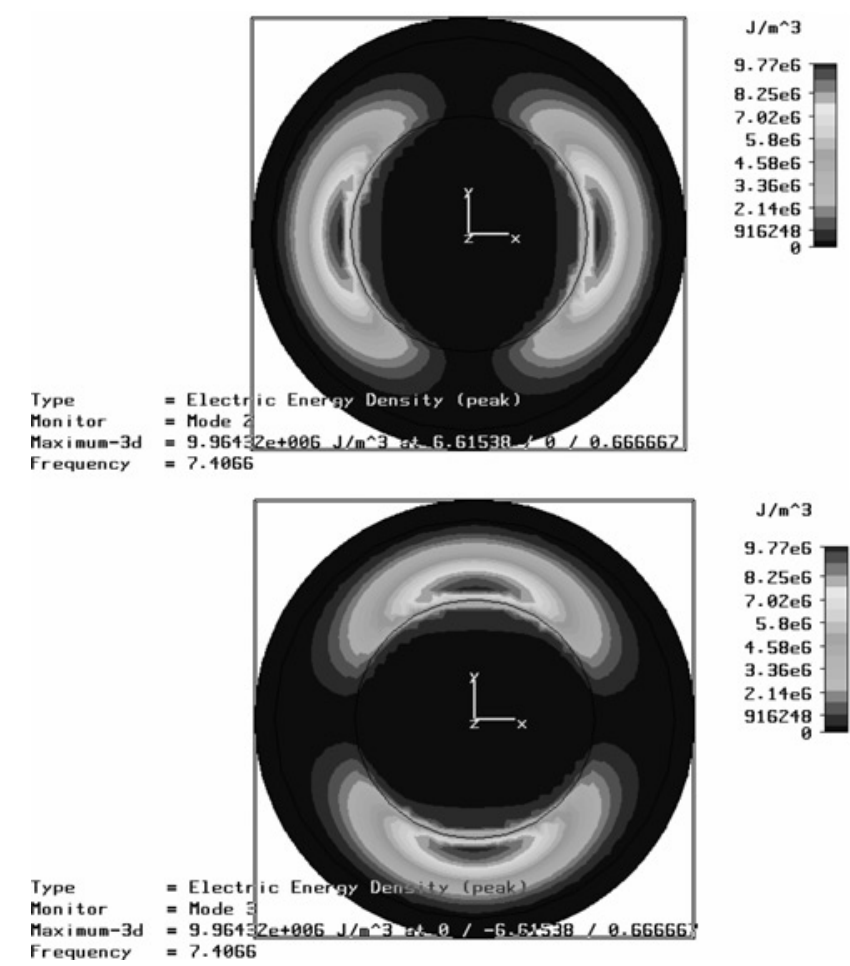

Figure 12 Electric energy densities in the transverse crosssection of the waveguide for the two polarisations of the two modes $E H_{11}$

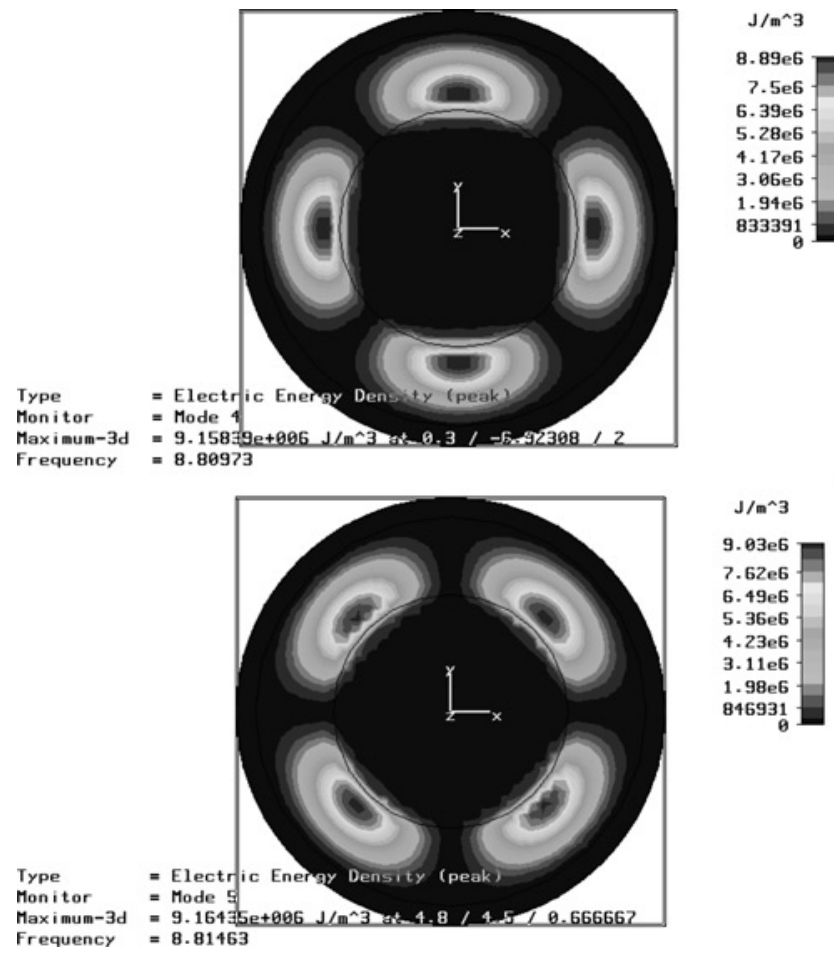

Figure 13 Electric energy densities in the transverse crosssection of the waveguide for the two polarisations of the two modes $\mathrm{EH}_{21}$

$r_{0}-r_{1}$ varies from 5 to $2 \mathrm{~mm}$ or $r_{1} / r_{0}$ varies between 0.5 and 0.2 ).

The width of the corrugation was kept at $1 \mathrm{~mm}$ as in the previous cases. We can again see the resonant phenomenon described in the previous section. It occurs at a highest ratio of $r_{1} / r_{0}$ of 0.76 , at which value, the mode becomes a forward wave and the left-handed propagation disappears.

It is possible to see that for some values of the corrugation and waveguide geometry, there exist, at the same frequency, both forward and backward wave modes, although with a different azimuthally symmetry. This is not only a

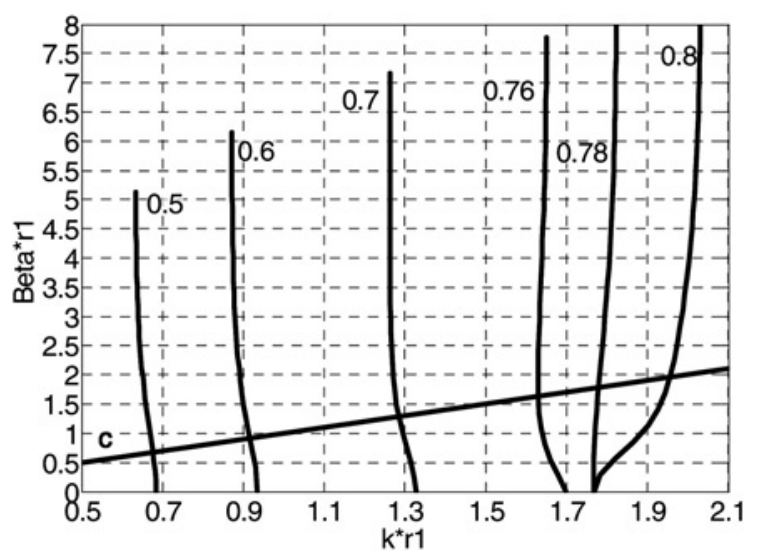

Figure 14 Parametric study of the mode $\mathrm{EH}_{11}$ for different values of the ratio $r_{1} / r_{0}$ 
phenomenon that occurs for the filled waveguide but also occurs in the unfilled case, as we can see in Fig. 7.

\section{Discussions}

In terms of applications, we propose taking advantage of the orthogonality of the propagating modes, for transmitting and receiving a signal in a waveguide at different group velocities. For example, with a receiver with a fixed sampling frequency, it is possible to have the signal carried by two separate orthogonal modes working at the same microwave frequency. One mode could be forward (right handed) and the other left handed. For example, consider the case for $r_{1} / r_{0}=0.48$ and $k r_{1}=1.7$, then $\beta r_{1}=0.63$ and 9.15 for which the two corresponding group velocities are $-0.16 c$ and +0.011 c, respectively (Fig. 15 ). The advantage of this is that the forward mode is slow propagating, whereas the left handed has a faster group velocity. Clearly, it is possible to transmit the same information on both modes. Then the receiver could get information at the sampling frequency from the forward mode. However, it could also extract information from the left-handed (faster group velocity) mode with apparent high frequency sampling. Therefore extra-information could be extracted from the left-handed mode wbich is not possible from the right-handed slower one.

Backward wave devices allow to obtain an index of refraction lower than one $(n<1)$. With this value, it is possible for the development of lenses with uniform thickness and index of refraction variable with distance from the centre, in order to make the exit plane a constantphase surface. The index of refraction should be stepped at intervals. Moreover, these lenses present advantages when used as arrays for wide scanning angles.

Corrugated circular waveguides have been used at millimetre wavelengths as band edge filters in Cosmic Microwave Background experiments with bolometric detectors (e.g. QUAD, Planck-HFI 2 radiotelescopes) [15, 19]. They are convenient in such systems as they allow both polarisations of the signal through from the corrugated horn antenna feed to the detector cavity without the need for the usual waveguide converters such as circular

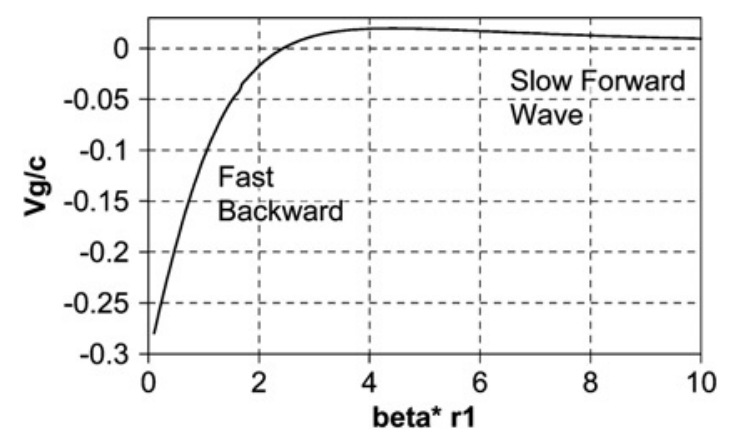

Figure 15 Showing how group velocity varies for large range of $\beta r_{1}$ for case of $r_{1} / r_{0}=0.48$ to rectangular. The lower band edge is thus sharply defined without the need for any extra filtering components. In the cases described in the literature, the waveguide is designed to propagate the lowest order $\mathrm{HE}_{11}$ mode. An alternative, of course, would be to use the backward propagating $\mathrm{EH}_{11}$ mode for cases where $r_{1} / r_{0}<0.48$. One advantage of this mode is both its upper and lower band edges are clearly defined in a region in which only one mode type can propagate, and a more compact waveguide structure can be used than that for the $\mathrm{HE}_{11}$ design. In any case, the disadvantage of $\mathrm{HE}_{11}$ design itself is that it also requires deep corrugations of $r_{1} / r_{0}<0.48$ otherwise two modes will be present which results in loss of coherence and an unpredictable antenna beam pattern $[16,19]$.

We have shown that we can achieve a left-handed behaviour without requiring complicated structures in a waveguide. For example, we can avoid the introduction of FSS inside the waveguide. Therefore the manufacture of the system is simpler and cheaper. We achieve left-handed propagation making use only of the corrugations, which are part of the structure of the guide. In addition, circular corrugated waveguide is simpler than the rectangular corrugated waveguide with filled corrugations on only one side reported in the literature $[8,9]$. Such a waveguide is more difficult to manufacture than the unfilled empty corrugated circular waveguide we have studied or even the filled one we have proposed.

\section{Conclusion}

We have analysed the left-handed propagation capabilities of infinite circular corrugated waveguides. A theoretical approach to the problem has been done following the surface impedance model for circular corrugated waveguides. The physical meaning of this kind of backward propagation has been related to resonances in the corrugated structure. Also the relationship between the power flow and group velocity for the waveguide is shown. The influence of the corrugations in the bandwidth and propagation characteristics of the different modes has been studied. We also realised a parametric analysis of the depth of the corrugations, with and without dielectric fill in the grooves, by using a full-wave simulator (CST). Several applications have been proposed for such structures.

The advantages of the circular corrugated waveguide are the easier manufacture compared with the rectangular waveguide even for the dielectric filled case, the availability of two polarisations for multiple applications and the miniaturisation of systems. Clearly, they are easier and cheaper to manufacture than those waveguides which use a complicated structure inside such as the FSS.

The future plans are to apply the surface impedance model to the dielectric filled guide, to explore applications with regard to lenses and filters, coupling, reflections, polarisation effects etc., and to analyse the possibility of 
transition sections for forward to backward propagation. Also, a close study of different depths of the corrugations along the waveguide is likely to bring useful results.

\section{Acknowledgment}

The authors thank the Universidad Carlos III de Madrid for the financial support given to this research and Prof. Leandro de Haro for his comments and review. Also, they thank for the very suitable and important comments of the reviewers of the journal.

\section{References}

[1] eleftheriades G.V., Balmain K.G.: 'Negative-refraction metamaterials' (Wiley, 2005, 1st edn.)

[2] CALOZ C., ITOH т.: 'Electromagnetic metamaterials: transmission line theory and microwave applications' (Wiley, Canada, Ltd., 2005, 1st edn.)

[3] elefheriades G.V., IYeR A.K., KReMer P.C.: 'Planar negative refractive index media using periodically L-C loaded transmission lines', IEEE Trans. Microw. Theory Tech., 2002, 50, pp. 2702-2712

[4] ELEFHERIADES G.V., SIDDIQUI O., IYER A.K.: 'Transmission line models for negative refractive index media and associated implementations without excess resonators', IEEE Microw. Comp. Lett., 2003, 13, pp. 51-53

[5] Marqués R., Mesa F., MARTel J., Medina f.: 'Comparative analysis of edge- and broadside-coupled split ring resonators for metamaterial design - theory and experiments', IEEE Trans. Antennas Propag., 2003, 51, (10), pp. 2572-2581

[6] hRABAR S., BARTOLIC J., SIPUS Z.: 'Waveguide miniaturization using uniaxial negative permeability metamaterial', IEEE Trans. Antennas Propag., 2005, 53, (1), pp. 110-119

[7] ESHRAH I.A., KISHK A.A., YAKOVLEV A.B., GLISSON A.W.: 'Spectral analysis of left-handed rectangular waveguides with dielectric-filled corrugations', IEEE Trans. Antennas Propag., 2005, 53, (11), pp. 3673-3683

[8] ESHRAH I.A., KISHK A.A., YAKOVLEV A.B., GLISSON A.W.: 'Rectangular waveguide with dielectric-filled corrugations supporting backward waves', IEEE Trans. Microw. Theory Tech., 2005, 53, (11), pp. 3298-3304

[9] KSHETRIMAYUM R., ZHU L.Z.: 'Guided-wave characteristics of waveguide based periodic structures loaded with various FSS strip layers', IEEE Trans. Antennas Propag., 2005, 53, (1), pp. 120-124

[10] CLARRICOATS P.J.B., OLVER A.D.: 'Corrugated horns for microwave antennas' (Peter Peregrinus Ltd, London, UK, 1984)

[11] OLVER A.D., CLARRICOATS P.J.B., KISHK A.A., SHAFAI L.: 'Microwave horns and feeds', IEE Electromagn. Waves Ser., 1994, 39, pp. 22-32

[12] eStebAN J., ReBollaR J.M.: 'Characterization of corrugated waveguides by modal analysis', IEEE Trans. Microw. Theory Tech., 1991, 39, (6), pp. 937-943

[13] AMARI S., VAHLDIECK R., BORNEMANN J., LEUCHTMANN P.: 'Spectrum of corrugated and periodically loaded waveguides from classical matrix eigenvalues', IEEE Trans. Microw. Theory Tech., 2000, 48, (3), pp. 453-460

[14] MURPhy J.A., COLGAN R., O'SUlLivan C., MAFFEI B., ADE P.: 'Radiation patterns of multi-moded corrugated horns for far-IR space applications', Infrared Phys. Technol., 2001, 42, pp. $515-528$

[15] GleESON E., MURPhy J.A., MAFFEl B., LANNIGAN W.: 'Corrugated waveguide band edge filters for $\mathrm{CMB}$ experiments in the far infrared', Infrared Phys. Technol., 2005, 46, pp. $493-505$

[16] Computer Simulation Technology, http://www.cst.com

[17] POZAR D.M.: 'Microwave Engineering' (Wiley, 1998, 2nd edn.)

[18] MAFFEI B., ADE P.A.R., TUCKER C.E., ET AL.: 'Shaped corrugated horns for cosmic microwave background anisotropy measurements', Int. J. Infrared Millim. Waves, 2005, 11, pp. 2023-2033

[19] GLEESON E., MURPHY J.A., MAFFEI B.: 'Phase centers of far infrared multi-moded horn antennas', Int. J. Infrared Millim. Waves, 2002, 23, (5), pp. 711-730 
Reproduced with permission of the copyright owner. Further reproduction prohibited without permission. 